\title{
Signatures of Adaptation and Acclimatization to Reef Flat and Slope Habitats in the Coral Pocillopora damicornis
}

\section{OPEN ACCESS}

Edited by:

Vianney Denis,

National Taiwan University, Taiwan

Reviewed by:

Rodrigo Carballo-Bolaños, National Taiwan University, Taiwan Kaho H. Tisthammer, University of Hawai'i at Manoa, United States

${ }^{*}$ Correspondence:

Shelby R. Marhoefer shelby.marhoefer@my.jcu.edu.au

Specialty section:

This article was submitted to Coral Reef Research

a section of the journal

Frontiers in Marine Science

Received: 03 May 2021

Accepted: 03 August 2021 Published: 03 September 2021

Citation:

Marhoefer SR, Zenger KR,

Strugnell JM, Logan M, van Oppen MJH, Kenkel $C D$ and

Bay LK (2021) Signatures of Adaptation and Acclimatization to Reef Flat and Slope Habitats in the Coral Pocillopora damicornis.

Front. Mar. Sci. 8:704709. doi: 10.3389/fmars.2021.704709

\section{Shelby R. Marhoefer ${ }^{1,2 *}$, Kyall R. Zenger ${ }^{2,3}$, Jan M. Strugnell/2,3,4, Murray Logan ${ }^{5}$, Madeleine J. H. van Oppen ${ }^{1,5,6}$, Carly D. Kenkel5,7 and Line K. Bay ${ }^{1,5}$}

'AIMS@JCU, Townsville, QLD, Australia, ${ }^{2}$ College of Science and Engineering, James Cook University, Townsville, QLD, Australia, ${ }^{3}$ Centre for Sustainable Tropical Fisheries and Aquaculture, James Cook University, Townsville, QLD, Australia, ${ }^{4}$ Department of Ecology, Environment and Evolution, School of Life Sciences, La Trobe University, Melbourne, VIC, Australia, ${ }^{5}$ Australian Institute of Marine Science, Townsville, QLD, Australia, ${ }^{6}$ School of BioSciences, The University of Melbourne, Parkville, VIC, Australia, ' Department of Biological Sciences, University of Southern California, Los Angeles, CA, United States

Strong population-by-habitat interactions across environmental gradients arise from genetic adaptation or acclimatization and represents phenotypic variation required for populations to respond to changing environmental conditions. As such, patterns of adaptation and acclimatization of reef-building corals are integral to predictions of the future of coral reefs under climate warming. The common brooding coral, Pocillopora damicornis, exhibits extensive differences in host genetic and microbial symbiont community composition between depth habitats at Heron Island in the southern Great Barrier Reef, Australia. An 18-month reciprocal field transplant experiment was undertaken to examine the environmental and genetic drivers behind variation in survival, weight gain, heat tolerance and algal symbiont community between the reef flat and slope habitats. We observed population-by-habitat interactions for in situ partial mortality and weight gain, where trait-related fitness of natives was greater than transplants in most cases, consistent with local adaptation. On average, flat colonies transplanted to the slope had a relatively low partial mortality but minimal weight gain, whereas slope colonies transplanted to the flat had relatively high partial mortality and average weight gain. Experimental heat tolerance was always higher in colonies sourced from the flat, but increased when slope colonies were transplanted to the flat, providing evidence of acclimatization in these colonies. The performance of certain slope to flat transplants may have been driven by each colony's algal symbiont (Symbiodiniaceae) community, and flat variants were observed in a small number of slope colonies that either had a fixed flat composition before transplantation or shuffled after transplantation. Host genotypes of previously identified genetic outlier loci could not predict survival following transplantation, possibly because of low sample size and/or polygenic basis to the traits examined. Local environmental conditions and Symbiodiniaceae composition may provide insight into the adaptive potential to changing environmental conditions.

Keywords: population-by-habitat interaction, local adaptation, acclimatization, reciprocal transplant, Pocillopora damicornis, trait-related fitness 


\section{INTRODUCTION}

Global surface temperatures are increasing, and climatic extremes are becoming more widespread and pronounced (Hansen et al., 2010; Cheng et al., 2019). This exposes whole ecosystems to conditions outside long-term baselines that result in severe stress and mortality across key species such as coral and kelp (Hughes et al., 2003; Wernberg et al., 2016; Smale, 2020). For these ecosystems to persist into the future, key habitat-forming species will either need to adapt, acclimatize or shift geographic range (Parmesan and Yohe, 2003; Burrows et al., 2011; Blois et al., 2013; Price et al., 2019). Adaptation arises when variation in fitness has a genetic basis and adaptive alleles can be selected for by local conditions over generations (Ellegren and Sheldon, 2008). Acclimatization is dependent upon an individual's potential for phenotypic plasticity, and can also be heritable and thus facilitates adaptation (Galloway and Etterson, 2007; Marshall, 2008; Putnam and Gates, 2015; Torda et al., 2017). For species with complex microbial communities, such as corals, the ability to respond to environmental change not only depends on the host, but also the adaptive and plastic potential of its symbiotic microorganisms (Chakravarti et al., 2017; Ziegler et al., 2017; Quigley et al., 2018). The ability of coral populations to adjust to multiple environmental conditions is important for long-term resilience, especially under rapidly changing climatic conditions. Therefore, comprehensive knowledge of the adaptive potential of species is required to understand and predict the future state of climate impacted ecosystems and best practice management (Van Hooidonk et al., 2016; Anthony et al., 2017; Hoegh-Guldberg et al., 2017).

Transplantation of organisms into environmental conditions that mimic climate scenarios predicted for their native habitat may uncover the likelihood of a species' persistence into the future. Reciprocal transplant experiments (RTEs) are used in evolutionary ecology to estimate the degree of genetic adaptation and plasticity that underpin organismal performance between local populations, where fitness-related traits are measured in individuals from two or more populations exposed to their distinct native and novel environments (Kawecki and Ebert, 2004; Hoeksema and Forde, 2008; Blanquart et al., 2013). Local adaptation is inferred when natives outperform foreign transplants (Blanquart et al., 2013; Savolainen et al., 2013), and acclimatization of a population manifests shifts in performance toward the locals in the novel environment and consequently outperforms its conspecifics that remained at the native environment (Kawecki and Ebert, 2004; Henn et al., 2018). The physiological responses of multiple populations to reciprocal transplantation may be coupled with genetic data to explore genotype-by-environment $(\mathrm{G} \times \mathrm{E})$ interactions (Blanquart et al., 2013; Drury and Lirman, 2021).

Diverse responses have been reported for corals reciprocally transplanted among various habitats. Local adaptation was evident in traits like survival, gene expression and Symbiodiniaceae community composition along environmental gradients such as depth, water quality and temperature. For example, coral species Acropora hyacinthus, Porites lobata, and Porites astreoides had divergent population phenotypes between environmentally variable and moderate habitats (Barshis et al., 2010; Palumbi et al., 2014; Bay and Palumbi, 2017; Kenkel and Matz, 2017). Reciprocal transplantation also revealed adaptive divergence among depth habitats in the coral species Seriatopora hystrix (Bongaerts et al., 2011), as well as the octocoral species Eunicea flexuosa (Prada and Hellberg, 2013) and Briareum asbestinum (West et al., 1993). Most recently, Acropora cervicornis genotypes reciprocally transplanted among numerous replicate reef sites with different thermal regimes in the Florida Reef Tract indicated $G \times$ E interactions for bleaching tolerance, where the greatest variability in bleaching tolerance among genotypes was within each site, and no genotype had superior bleaching tolerance in all transplant locations (Drury and Lirman, 2021). Studies of reciprocal transplantation of corals continue to reveal that performance is affected by environmental conditions, but varies significantly by colony, highlighting the need to expand replicate RTEs among small spatial scales to elucidate colony differences.

The reefs in the Capricorn Bunker Group in the southern Great Barrier Reef, Australia, provide an ideal study system to test for local adaptation. Many reefs in this region have well-developed reef crests and ponding lagoons that lead to distinct habitats across small spatial scales (Takabayashi and Hoegh-Guldberg, 1995; van Oppen et al., 2018). For example, lagoon reef flat habitats have more variable and extreme water temperature and light irradiance compared to the slope at Heron Island (van Oppen et al., 2018). Despite being within the dispersal distance for most coral larvae including brooding species, Pocillopora damicornis were found to be genetically, physiologically, and morphologically partitioned among depth habitats at Heron Island (Takabayashi and Hoegh-Guldberg, 1995; van Oppen et al., 2018) [similar patterns exist for P. damicornis populations at Kāne'ohe Bay, Hawai'i (Gorospe and Karl, 2015)]. Sixteen host associated genetic outlier loci distinguished slope and flat populations at Heron Island and communities of dinoflagellate photosymbionts and prokaryotes also diverged between habitats (van Oppen et al., 2018). The present evidence of divergence between colonies that originated from the flat or slope supports the potential for local adaptation to these habitats, although it is unknown if and how these differences affect physiological performance under novel and stressful conditions.

To further understand the relative importance of acclimatization and adaptation of reef building corals to increasing and more extreme environments, we used P. damicornis colonies from Heron Island in a two-part experiment consisting of a replicated RTE across depth habitats and a common garden stress test. In particular, we were interested in testing whether the genetic differences unveiled in van Oppen et al. (2018) were reflected in phenotypes and to tease apart the relative contribution of genetic adaptation versus acclimatization of phenotypic traits. We measured three vital traits over 18 months in the field, and then measured relative heat tolerance by exposing the surviving colonies to 14 days of ex 
situ experimental heat stress. The resulting information provides clarity to the influence of environmental selection on trait-related fitness of populations within a close spatial proximity and better serves as a reference for the management of coral species.

\section{MATERIALS AND METHODS}

\section{Experimental Design}

Replicate RTEs were carried out between two depth habitats (reef flat and slope) and at two adjacent reef sites at Heron Island between March 2012 - August 2013. Experiments were carried out using the source colonies of $P$. damicornis in van Oppen et al. (2018) and collected under Great Barrier Reef Marine Park Authority permit G12/34752.1. The flat and the slope habitats sampled here are separated by less than $100 \mathrm{~m}$ in horizontal distance and approximately $5 \mathrm{~m}$ in depth at each of the two replicate sites, Canyons and Gardens. These reef sites are separated by $\sim 1 \mathrm{~km}$ (Canyons flat: $23^{\circ} 27^{\prime} 14.50^{\prime \prime} \mathrm{S}$, $151^{\circ} 55^{\prime} 34.55^{\prime \prime} \mathrm{E}$; Canyons slope: $23^{\circ} 27^{\prime} 18.80^{\prime \prime} \mathrm{S}, 151^{\circ} 55^{\prime} 28.29^{\prime \prime} \mathrm{E}$; Gardens flat: $23^{\circ} 26^{\prime} 48.50^{\prime \prime} \mathrm{S}, 151^{\circ} 54^{\prime} 46.34^{\prime \prime} \mathrm{E}$; Gardens slope: $\left.23^{\circ} 26^{\prime} 50.54^{\prime \prime} \mathrm{S}, 151^{\circ} 54^{\prime} 54.46^{\prime \prime}\right)$. Over the 18 -month experiment, mean annual temperature did not vary between habitats, but the range did, with colonies on the flat experiencing temperatures between 15.8 and $33.2^{\circ} \mathrm{C}$, while colonies on the slope experienced temperatures between 18.7 and $28.0^{\circ} \mathrm{C}$ (van Oppen et al., 2018). Ambient light (measured as photosynthetic active radiation) also varied annually and was three times higher and four times more variable in the flat versus the slope (flat: $150-1,400 \mu \mathrm{mol}$ photons $\mathrm{m}^{-2} \mathrm{~s}^{-1}$; slope: $100-400 \mu \mathrm{mol}$ photons $\mathrm{m}^{-2} \mathrm{~s}^{-1}$ ).

At each site and habitat, 23 - 26 colonies were collected (total $n=97)$ and fragmented into two halves. Within a day, one half of each colony was placed onto experimental racks in its habitat of origin, and its genetic clone was transplanted to the reciprocal habitat within each site, resulting in four treatment groups replicated at two sites: flat to flat (FF), flat to slope (FS), slope to flat (SF), and slope to slope (SS). At each site, two $2 \mathrm{~m}$ by $2 \mathrm{~m}$ wire mesh racks were erected approximately $0.5 \mathrm{~m}$ above the substrate, and fragmented coral colonies were attached in a haphazard manner across these racks with cable ties. At each sampling time, colonies were removed from the racks, sampled and measured, then reattached to the rack.

\section{Physiological and Genetic Measurements}

\section{Partial Colony Mortality and Cumulative Weight Gain}

Partial colony mortality and weight gain of coral colonies were measured in the field over four time points in March and August of 2012 and 2013. Colonies were visually scored for partial mortality to the nearest 5\% using the Coral Watch health chart as a reference (Siebeck et al., 2006). Wet buoyant weight was also obtained underwater using calibrated OHAUS Triple Beam 700 series balancing scales. Any single colony with less than $100 \%$ mortality was considered to be alive. Percent cumulative buoyant weight gain was calculated using the first and final time points [(current weight - initial weight)/initial weight]. Small samples $(\sim 5 \mathrm{~g}$ and $<7 \mathrm{~cm})$ taken for DNA analysis underwater were later weighed and added to the weight gain at that time point.

\section{Heat Tolerance}

At the end of the field transplant experiment on 17 August 2013, the remaining living colonies were transported under flow-through conditions aboard the RV Cape Ferguson to the Australian Institute of Marine Science headquarters in Townsville where they were kept first in outdoor $1000 \mathrm{~L}$ tanks for 10 days. On 27 and 28 August 2013, they were further fragmented into single branches then acclimatized in indoor $30 \mathrm{~L}$ flow-through tanks at $24^{\circ} \mathrm{C}$ conditions. Depending on the amount of live tissue per colony remaining, there were between 3 and 20 replicate fragments per colony for each level of heat stress: $24^{\circ} \mathrm{C}, 29^{\circ} \mathrm{C}, 30^{\circ} \mathrm{C}, 31^{\circ} \mathrm{C}$ (Supplementary Table 1). Water temperatures were ramped up from ambient $\left(24^{\circ} \mathrm{C}\right)$ to its designated temperature within 1 day on 1 October 2013, and thereafter maintained at these levels for 13 days, for a total of 14 days of heat stress. Lights were sustained under a slightly shifted 12:12 light-dark cycle at a constant level of $250 \mu \mathrm{mol}$ photons $\mathrm{m}^{-2} \mathrm{~s}^{-1}$. Heat tolerance was quantified by proxy of the maximum quantum yield (Fv/Fm), measured with Pulse-Amplitude Modulation (PAM) fluorometry using a diving PAM (Walz) with default settings. Fragmented colonies were measured at the end of their dark cycle before experimental dawn at 10:00AM.

\section{Coral Host Outlier Loci Analysis}

We analyzed the genotype frequencies of 16 highly significant single nucleotide polymorphism (SNP) outliers from 13 loci identified by BayeScan (Foll, 2012) and Fdist between habitat in van Oppen et al. (2018). If genotypes of outlier loci were closely linked to survival, we anticipated the genotype frequencies of the survivors to resemble the local habitat it was transplanted into. To identify differences in allelic frequencies among site and treatment locations, pairwise $F_{\mathrm{ST}}$ and their corresponding $p$-values on the 16 outlier loci between the survivors were calculated in ARLEQUIN v3.5.2.2 (Excoffier and Lischer, 2010). $F_{\text {ST }}$ tests were done with and without sites combined for the four treatment groups. As considerable mortality occurred between March and August 2013, the March 2013 time point (12 months post-transplantation) was used to maximize the number of survivors per treatment and increase the power of the analysis (Table 1).

\section{DNA Extraction, ITS2 Sequencing and Assignment}

We extracted DNA from samples at two time points, FF and SS treatments in April 2012 and all treatment groups in March 2013 (Supplementary Table 2). The native treatment group samples were taken 1 month after initial transplantation due to a mild bleaching response in the SF colonies immediately following the transplantation in March 2012. Following Wayne's method (Wilson et al., 2002), pieces of coral tissue and skeleton previously stored at $-80^{\circ} \mathrm{C}$ were vortexed in a fresh 
TABLE 1 | The number of survivors (n), average partial mortality $(P M) \pm S E$, and average cumulative weight gain (WG) \pm SE per site and treatment group at each sampling time point.

\begin{tabular}{|c|c|c|c|c|c|c|c|}
\hline Treatment group & Date & Canyons survivors (n) & Gardens survivors (n) & Canyons PM & Gardens PM & Canyons WG & Gardens WG \\
\hline \multirow[t]{4}{*}{ FF } & March-12 & 24 & 26 & $0.00 \pm 0.00$ & $0.00 \pm 0.00$ & - & - \\
\hline & August-12 & 24 & 26 & $0.00 \pm 0.00$ & $9.31 \pm 4.16$ & $38.97 \pm 3.64$ & $44.40 \pm 4.28$ \\
\hline & March-13 & 23 & 22 & $3.70 \pm 1.34$ & $45.42 \pm 9.65$ & $111.80 \pm 10.93$ & $82.40 \pm 11.93$ \\
\hline & August-13 & 23 & 18 & $4.35 \pm 1.52$ & $56.77 \pm 11.37$ & $145.39 \pm 11.93$ & $116.00 \pm 19.01$ \\
\hline \multirow[t]{4}{*}{ FS } & March-12 & 24 & 26 & $0.00 \pm 0.00$ & $0.00 \pm 0.00$ & - & - \\
\hline & August-12 & 22 & 26 & $7.18 \pm 4.24$ & $25.15 \pm 6.06$ & $31.57 \pm 2.55$ & $2.18 \pm 3.17$ \\
\hline & March-13 & 21 & 10 & $19.96 \pm 7.85$ & $84.52 \pm 8.78$ & $48.80 \pm 6.79$ & $-6.36 \pm 7.89$ \\
\hline & August-13 & 20 & 7 & $24.91 \pm 8.80$ & $88.00 \pm 9.62$ & $69.93 \pm 8.79$ & $19.04 \pm 7.27$ \\
\hline \multirow[t]{4}{*}{ SF } & March-12 & 24 & 24 & $0.00 \pm 0.00$ & $0.00 \pm 0.00$ & - & - \\
\hline & August-12 & 23 & 24 & $26.88 \pm 6.83$ & $13.96 \pm 6.07$ & $18.02 \pm 2.65$ & $19.70 \pm 2.10$ \\
\hline & March-13 & 13 & 5 & $77.38 \pm 9.53$ & $88.29 \pm 12.41$ & $29.21 \pm 7.84$ & $41.69 \pm 22.07$ \\
\hline & August-13 & 3 & 3 & $91.67 \pm 15.87$ & $95.38 \pm 11.79$ & $76.84 \pm 12.12$ & $91.91 \pm 47.00$ \\
\hline \multirow[t]{4}{*}{ SS } & March-12 & 24 & 23 & $0.00 \pm 0.00$ & $0.00 \pm 0.00$ & - & - \\
\hline & August-12 & 24 & 23 & $0.08 \pm 0.08$ & $1.30 \pm 0.65$ & $43.04 \pm 3.86$ & $25.89 \pm 1.66$ \\
\hline & March-13 & 24 & 22 & $15.96 \pm 6.70$ & $21.74 \pm 5.82$ & $97.14 \pm 11.06$ & $56.47 \pm 5.24$ \\
\hline & August-13 & 20 & 19 & $24.38 \pm 8.90$ & $31.74 \pm 9.09$ & $128.67 \pm 15.19$ & $82.46 \pm 8.26$ \\
\hline
\end{tabular}

FF, flat to flat; FS, flat to slope; SF, slope to flat; SS, slope to slope.

grinding buffer and processed using a salting-out protocol. The resulting pellet was washed with $70 \%$ ethanol, then resuspended in $70 \mu \mathrm{L}$ milli-Q water and stored at $-20^{\circ} \mathrm{C}$. The quality of DNA was checked using a NanoDrop NDTND-1000 and electrophoresis, then diluted to 1:10 in Milli-Q water. The ITS2 locus was amplified with PCR with negative controls, using AmpliTaq Gold 360 Master Mix and the following primers: ITS2-F (5'-GTGAATTGCAGAACTCCGTG-3') and ITS2-R ( $5^{\prime}$ CCTCCGCTTACTTATATGCTT-3'). PCR was performed in a total volume of $30 \mu \mathrm{L}$ per sample, with $2 \times$ AmpliTaq Gold Master Mix, 1.2 $\mu \mathrm{L}$ of each $10 \mu \mathrm{M} \mathrm{F}$ and R primer, 9.6 $\mu \mathrm{L}$ PCR-grade water, and $3 \mu \mathrm{L}$ of DNA dilution, nominating the final primer concentrations as $0.4 \mu \mathrm{M}$. The PCR consisted of $10 \mathrm{~min}$ at $95^{\circ} \mathrm{C}$ of initial denaturation, then 30 cycles of $30 \mathrm{~s} 95^{\circ} \mathrm{C}$ denaturation, $60 \mathrm{~s} 57^{\circ} \mathrm{C}$ annealing, and $30 \mathrm{~s} 72^{\circ} \mathrm{C}$ extension. Final elongation was for $7 \mathrm{~min}$ at $72^{\circ} \mathrm{C}$ and held at $4^{\circ} \mathrm{C}$. The PCR product was checked for correct amplicon size and yield on $2 \%$ agarose gel at $90 \mathrm{~V}$ for $60 \mathrm{~min}$ and shipped cold to Ramaciotti Centre for Genomics (University of New South Wales, Sydney, NSW, Australia) for sequencing on the Illumina MiSeq platform. Raw sequences are available on NCBI Sequence Read Archive under Accession no. PRJNA741589. Demultiplexed paired fastq.gz ITS2 files on Illumina were processed through the SymPortal.org database (Hume et al., 2019). SymPortal utilizes Mothur and minimum entropy decomposition to assign sequences and taxon type, with intragenomic groupings based on $97 \%$ similarity. Sequences from the samples that matched the SymPortal online database are identified as defining intragenomic variants (DIVs), which were analyzed here, and hereon referred to as "variants".

\section{Statistical Analysis}

All statistical analyses were carried out in R v4.0.2 (R Core Team, 2020). The three physiological traits (partial colony mortality, cumulative weight gain, and heat tolerance) were evaluated using generalized linear mixed effects models with the glmmTMB package (Brooks et al., 2017). Partial mortality and heat tolerance were modeled against the beta family with the logit link function because the data values were between 0 and 1 . Weight gain was modeled against the Gaussian family because the data were a continuous measurement with some negative values. The model for partial mortality and weight gain included site (Canyons, Garden), source (flat, slope) and transplant (flat, slope) locations as fixed effects, and colony genetic identity as a random effect. Partial mortality and weight gain were both analyzed for the final time point (August 2013). Because of reduced numbers of survivors at the end of the field transplant experiment, heat treatment effects were combined by site and examined between flat and slope habitats only. Thus, the model for heat tolerance included temperature $\left(24^{\circ}, 29^{\circ}, 30^{\circ}, 31^{\circ} \mathrm{C}\right)$, source and transplant locations as fixed effects, and colony genetic identity as a random effect. Wald's Chi-square Tests were implemented on the glmmTMB models and were set to type III based on significant interaction terms. Contrasts were investigated with pairwise estimated marginal mean trait-related fitness with the emmeans package (Lenth et al., 2019) to make population-level predictions based on the small sample size for each treatment. The $p$-values for contrasts were adjusted using Holm's method for all models, but with 16 comparisons for partial mortality and cumulative weight gain models, and 24 comparisons for the heat stress model.

Symbiodiniaceae community data were trimmed to include sequence variants that were represented with $>0.005 \%$ total abundance and samples with $>20,000$ reads. The trimmed community data plus the sample data frame were combined in Phyloseq (McMurdie and Holmes, 2013) and were tested for permutational multivariate analysis of variance using the Vegan package (Oksanen et al., 2019) on Bray-Curtis distances with 999 permutations, and subsequent pairwise comparisons 
with Holm's method of adjustments for 16 ecologically relevant comparisons. Analysis of dispersion with 999 permutations were run for comparisons that had significantly different variance. The community composition of the habitats (flat vs. slope) was compared between the sampling years (April 2012 and March 2013), and all other tests were performed on treatment groups (FF, FS, SF, SS) with samples and data collected in March 2013.

Multivariate analysis of variance with 999 permutations were also used to test for associations between Symbiodiniaceae community composition with partial mortality and cumulative weight gain among source and transplant habitats with data from March 2013. These tests were run separately per site to prevent false interpretation of results. Heat tolerance was not assessed for an association with community composition given the lack of replicate samples per treatment group that had both genetic data and yield data (Supplementary Tables 1, 2). Specifically, there were six total SF colonies $(258,262,267,453,455,471)$ that had Symbiodiniaceae composition data and three SF colonies $(267,458,464)$ that were further fragmented and exposed to the heat stress experiment at $31^{\circ} \mathrm{C}$. Permutational analysis of dispersion tests were not able to be conducted for associations of Symbiodiniaceae composition and trait physiologies due to lack of complexity in the R function.

\section{RESULTS}

\section{Partial Colony Mortality}

Whole colony mortality was observed in all treatment groups, especially in transplant treatments, resulting in uneven sample sizes (Table 1). Partial mortality of colonies in August 2013 was affected by the interaction between source and transplant habitats, i.e., treatment groups pooled by replicate reef sites (Wald's Chisq = 18.31, $p<0.0001$; Supplementary Table 3), and between source and site averaged over the transplant habitats (Wald's Chisq $=6.15, p=0.013$ ). Slope-sourced colonies in both habitats showed similar levels of partial mortality between sites, but the flat-sourced colonies at the Gardens site had higher partial mortality in both habitats than at the Canyons site (Figure 1A). The partial mortality of colonies in the SF treatment group was two times greater than their SS counterparts and followed initial bleaching of this treatment group. At the Canyons, the partial mortality of the FS treatment group was not significantly different from the SS treatment group at the habitat transplanted into, or the FF treatment group in the habitat of origin. At the Gardens, the partial mortality of FS treatment group was $20 \%$ greater than their FF counterparts, though not significant, and 35\% greater than the SS treatment group (estimated marginal means Adj. $p=0.0048$; Supplementary Table 4). Between the two sites, the FS treatment group had $43 \%$ greater partial mortality at the Gardens than at the Canyons (estimated marginal means Adj. $p<0.0001$ ).

\section{Cumulative Weight Gain}

The difference in cumulative weight gain in colonies after 18 months of transplantation (March 2013) was significant for the interaction between source and transplant habitats

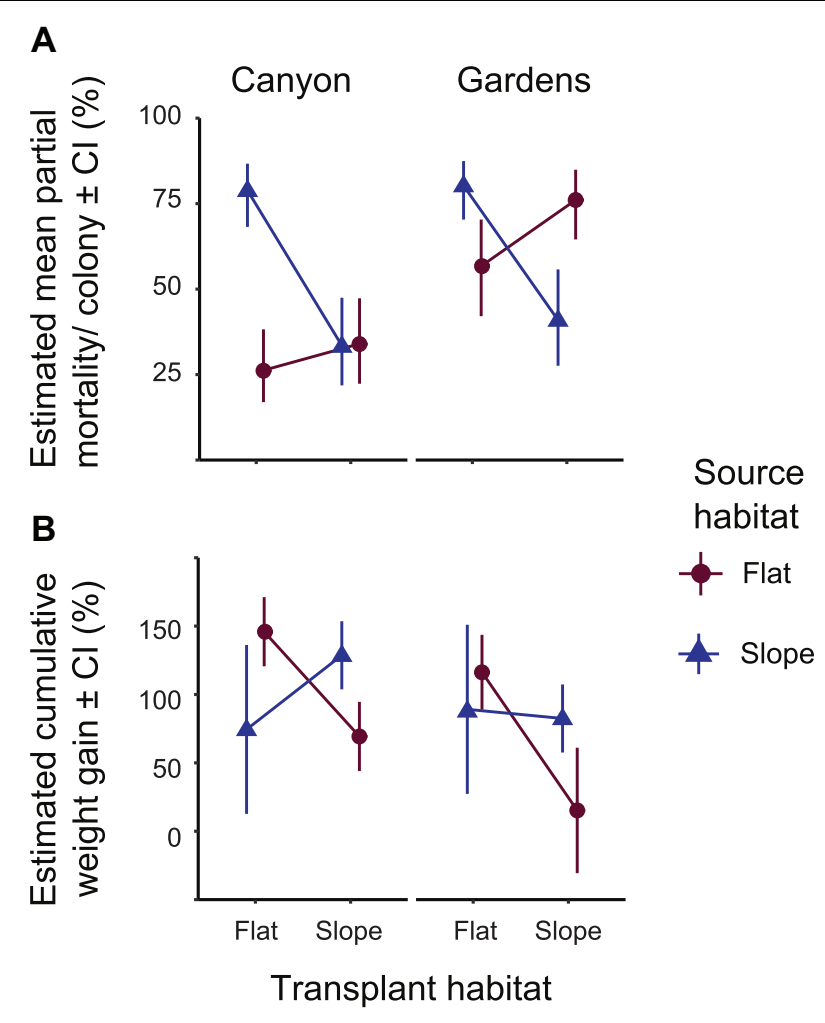

FIGURE 1 | Estimated means of (A) percent partial colony mortality and (B) percent cumulative weight gain per colony after 18 months in the field (August 2013). Positive numbers designate weight gain, negative numbers designate weight loss. Error bars on both $(\mathbf{A}, \mathbf{B})$ indicate range of possible means with 95\% confidence. Significance tables, Supplementary Table 4 accompanies (A) and Supplementary Table 6 accompanies (B).

(Wald's Chisq $=13.00, p=0.0003$; Supplementary Table 5), and insignificant between sites. The mean percent cumulative weight gain per colony in the FS treatment group was only $69 \%$ greater than its initial weight at the Canyons and 15\% greater than its initial weight at the Gardens (Figure 1B). Some FS colonies at the Gardens had up to $30 \%$ cumulative weight loss, demonstrated by the confidence interval reaching negative values. Transplants from the SF treatment group had highly variable weight gain as seen in the large range in confidence from the estimated means at both sites. At the Canyons, the SF treatment group had significantly lower estimated weight gained compared to their SS counterparts (estimated marginal means Adj. $p>0.0001$; Supplementary Table 6) and the FF treatment group at the habitat transplanted into (estimated marginal means Adj. $p>0.0001$ ). Conversely, at the Gardens, the cumulative weight gained in the SF treatment group was $6.6 \%$ greater, but not significantly different, than their SS counterparts, and was also not significantly lower than the FF treatment group (Figure 1B).

\section{Heat Tolerance}

Heat tolerance was measured using the maximum quantum yield $(\mathrm{Fv} / \mathrm{Fm})$ as a proxy. Fv/Fm declined with increasing 

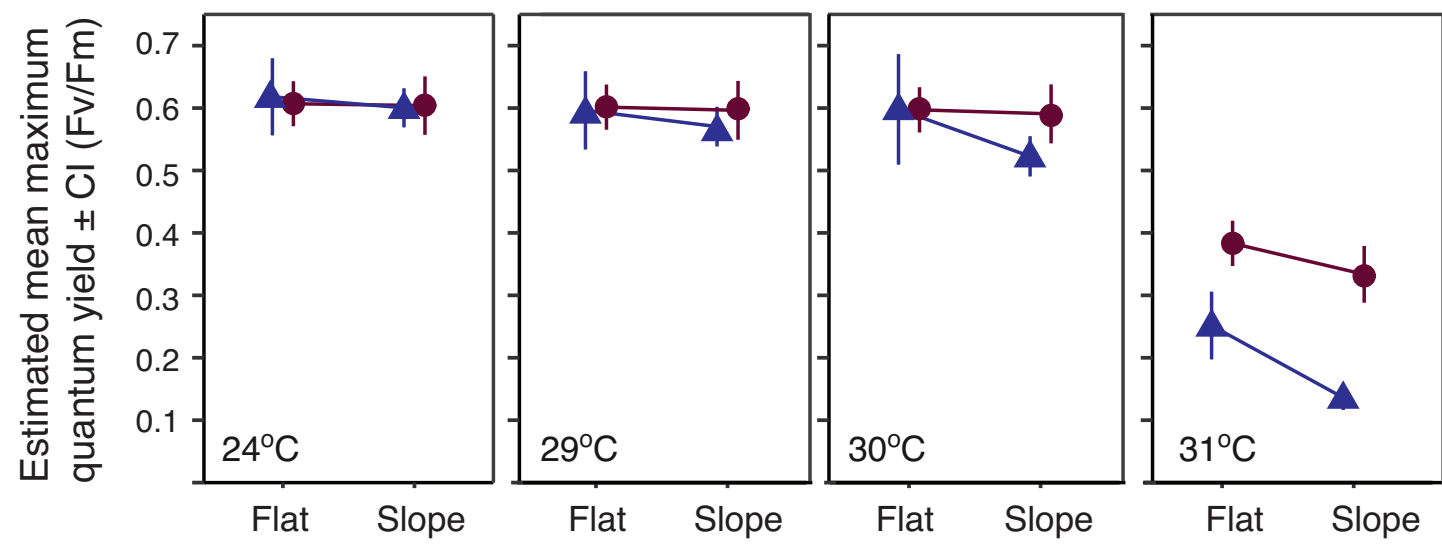

\section{Source habitat}

\section{Transplant habitat}

FIGURE 2 | Estimated mean maximum quantum yield \pm SE after 14 days of ex situ heat stress at ambient and elevated temperatures, post 18-month reciprocal transplant. Error bars indicate range of possible means with 95\% confidence. Significance table, Supplementary Table 8, accompanies this figure.

temperature (Figure 2) and was significantly affected by the interaction between temperature condition and source habitat (Wald's Chisq $=11.03, p=0.012$; Supplementary Table 7). At $30^{\circ} \mathrm{C}$, the maximum quantum yield of the $\mathrm{FF}$ treatment group was significantly higher than that of the SS treatment group (estimated marginal means Adj. $p=0.010$ ). At $31^{\circ} \mathrm{C}$, a large drop in maximum quantum yield was observed for all treatment groups. Flat-sourced treatment groups (FF and FS) were not significant from one another, and had between 13 and $20 \%$ greater yield values than the slope-sourced treatment groups (SS and SF), regardless of transplant habitat. The yield for colonies in the SF treatment group was $11.5 \%$ greater than the SS treatment group (estimated marginal means Adj. $p=0.0003$; Supplementary Table 8). All pairwise comparisons for temperature conditions at 24 and $29^{\circ} \mathrm{C}$ were not significantly different.

\section{Coral Host Genetics}

Outlier SNP pairwise $F_{\text {ST }}$ values between surviving colonies of the Gardens and Canyons sites indicated that there was no genetic differentiation between site replication treatments (i.e., FF, SS, FS, and SF at each location), whereby all $F_{\text {ST }}$ values where not significantly different from zero and the genetic composition of the replicated treatment groups between the sites could be considered the same (Supplementary Table 9). When combining treatment group data, pairwise $F_{\text {ST }}$ averaged over all 16 outliers support the resemblance of transplant treatment groups to colonies sourced from their native rather than novel habitat (Supplementary Figure 1) as averaged $F_{\mathrm{ST}}$ values between native and transplant treatment groups were not significantly different from zero (flat-sourced: -0.012 and slope-sourced: -0.042 ; Table 2). Other pairwise comparisons between transplant group and the natives at the novel habitat had high $F_{\mathrm{ST}}$ values (range $0.491-0.531$ ) and statistical significance $(p=0.000)$ indicating robust allelic differentiation. All combined site differentiation patterns were also directly replicated in the individual site comparison data (Supplementary Table 9).

\section{Symbiodiniaceae Dynamics}

A quality-controlled dataset yielded a total of 123 Symbiodiniaceae ITS2 sequence variants in 159 samples, $100 \%$ of which were from the genus Cladocopium. The majority of the Cladocopium variants were C42 in flat-sourced colonies, C33 in slope-sourced colonies, and C1 throughout (Figure 3A). Permutational multivariate analysis of variance results confirmed that samples from the native FF and SS treatment groups were distinct $\left(F_{1,127}=345.86, p=0.001\right.$; Supplementary Table 10 $)$ and did not statistically change over time $\left(F_{1,127}=2.47, p=0.110\right)$. The Symbiodiniaceae community composition was significantly affected by the interaction of site, source and transplant location, as in site by treatment group (permutational MANOVA: $F_{1,70}=3.470, p=0.039$; Supplementary Table 11), and was not significantly dispersed for the same interaction (permutational analysis of dispersion; Supplementary Table 12).

Pairwise comparisons of permutational multivariate analysis of variance revealed that FS colonies did not change after

TABLE 2 | Pairwise $F_{S T}$ values (bottom diagonal) and $F_{S T} p$-values (top diagonal) between surviving corals in all treatment groups among both sites 12 months after reciprocal transplantation (March 2013) for 16 single nucleotide polymorphism (SNP) outliers.

\begin{tabular}{lcccc}
\hline Treatment group & FF & SS & FS & SF \\
\hline FF & - & $\mathbf{0 . 0 0 0}$ & 0.892 & $\mathbf{0 . 0 0 0}$ \\
SS & 0.501 & - & $\mathbf{0 . 0 0 0}$ & 0.942 \\
FS & -0.012 & 0.531 & - & $\mathbf{0 . 0 0 0}$ \\
SF & 0.491 & -0.042 & 0.516 & -
\end{tabular}

Bold $p$-values indicate statistical significance. FF, flat to flat; FS, flat to slope; SF, slope to flat; SS, slope to slope. 


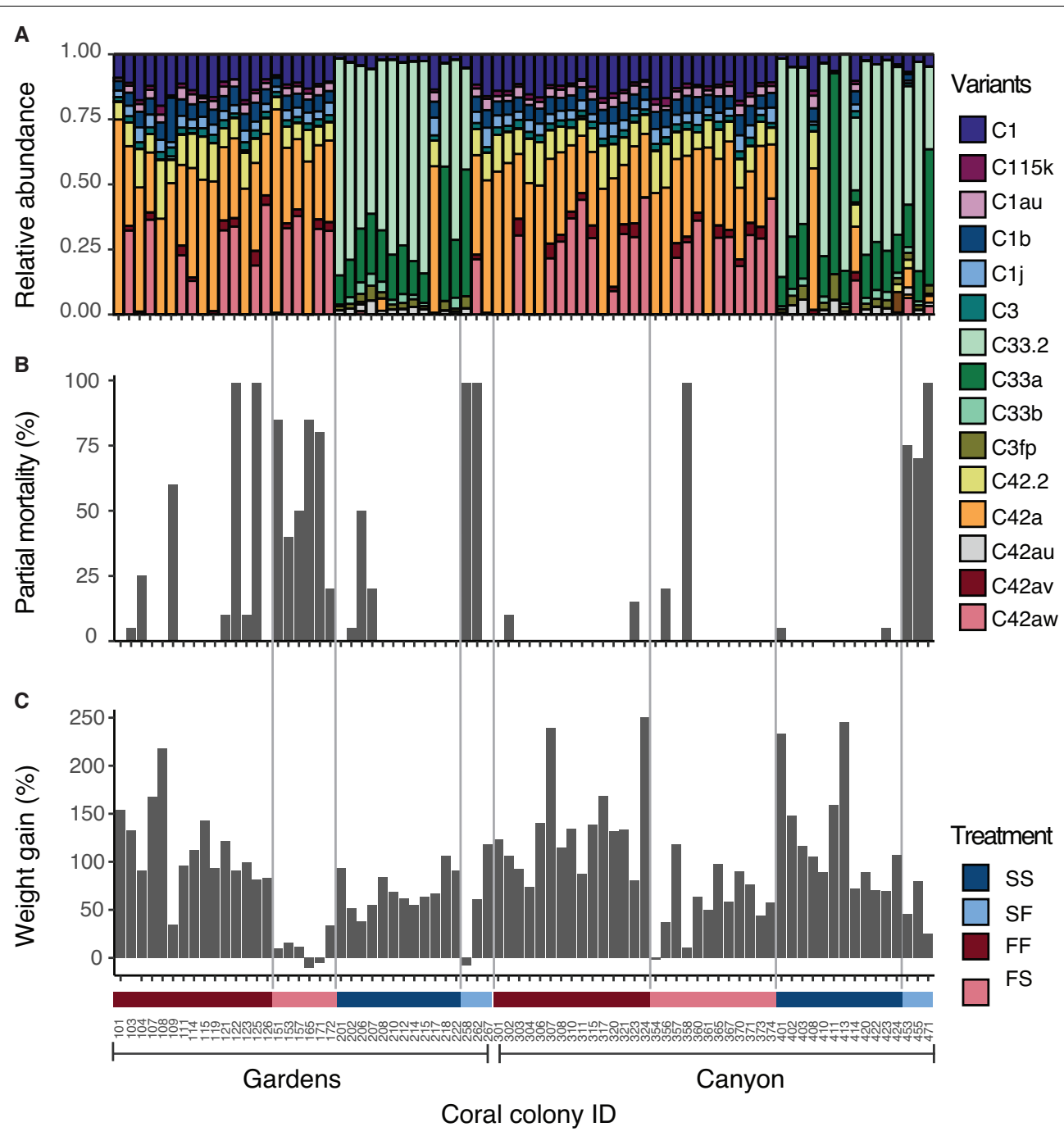

FIGURE 3 | Responses of individual coral colonies organized by site and treatment group after 12 months of reciprocal transplantation (March 2013 ). Numbers on the $X$-axis are colony ID and each ID number may correspond with a matching genet in the opposite habitat (i.e., FF ID 101 matches FS ID 151). All matching genetic pairs are listed in Supplementary Table 16. (A) Symbiodiniaceae community in coral tissue. Each color denotes relative abundance of different ITS2 sequence variants present. (B) Percent partial colony mortality. (C) Percent cumulative weight gain. Positive numbers designate weight gain, negative numbers designate weight loss.

transplantation, exhibited by statistical significance from the SS colonies (Canyons: Adj. $p=0.014$; Gardens: Adj. $p=0.026$; Supplementary Table 13), and none of them harbored sequence variants from the slope (Figure 3A). At the Canyons site, the colonies in the SF treatment group primarily maintained slope variants, and had significantly different community composition from the native FF colonies (Adj. $p=0.024$; Supplementary Table 13), but two of these three colonies harbored a low abundance of flat variants. One Canyons SF colony increased abundance of flat variants after transplantation (SF ID 453 and SS ID 403), and one could not be compared to its SS counterpart due to loss of samples after the experiment (ID 471). In contrast, at the Gardens, two of the three SF colonies were completely dominated by flat, C42 variants (Figure 3A), and were not statistically different from the FF treatment group
(Supplementary Table 13). Of these two Gardens SF colonies, one already maintained the flat variants in the SS treatment group (ID 267/217), while the other changed after transplantation into the flat habitat (ID 262/212).

Overall, the Symbiodiniaceae community composition 12 months after transplantation was not associated with the percent of partial mortality per colony at either site (permutational MANOVA Canyons: Supplementary Table 14, Gardens: Supplementary Table 15; Figure 3B), but was associated with the interaction of percent cumulative weight gain and transplant habitat at the Gardens site (permutational MANOVA Gardens: $F_{1,35}=4.817, p=0.015$; Supplementary Table 15). Typically, there was greater weight gain for colonies that harbored flat variants at the flat habitat and lesser weight gain with flat variants at the slope habitat (Figure $3 \mathrm{C}$ ). The 
SF colonies at the Gardens site that were comprised of flat Symbiodiniaceae variants had weight gain comparable to colonies in their native habitat. Further, the Gardens SF colony (ID 267) that maintained flat variants prior to transplantation had greater weight gain than most of the slope colonies at the slope, and also exhibited $0 \%$ mortality. Although heat tolerance was not tested statistically against Symbiodiniaceae composition, SF ID 267 had higher heat tolerance than its matching SS ID 217 , with an average yield value $(\mathrm{Fv} / \mathrm{Fm}) \pm$ standard error of $0.37 \pm 0.02$ and $0.34 \pm 0.07$, respectively.

\section{DISCUSSION}

Lower partial mortality and greater weight gain in individuals living in native versus non-native habitats confirmed strong local adaptation to depth habitat across small spatial scales in $P$. damicornis colonies from Heron Island as proposed by Takabayashi and Hoegh-Guldberg (1995) and van Oppen et al. (2018). We also detected signatures of adaptation and acclimatization to heat stress. Native reef flat colonies were more tolerant of elevated temperatures, regardless of transplantation location, demonstrating adaptation, and slope colonies transplanted to the flat increased heat tolerance compared to slope colonies that stayed at the slope, showing acclimatization. The importance of the Symbiodiniaceae symbionts for coral holobiont physiological trait-related fitness was evident from the significant association between the presence of a "flat" Symbiodiniaceae community and an increase in weight gain in a small number of slope colonies following transplantation to the flat at the Gardens site. Variation in physiological performances, host genotypes and symbiont communities between colonies native to the flat or slope habitat are characteristic of local adaptation and indicates that these colonies should be managed as separate populations despite their close physical proximity.

Physiological performance of $P$. damicornis colonies in the Capricorn Bunker Island group may be reliably predicted based on the environmental regimes in the habitat of origin, similarly to Porites lobata in American Samoa (Barshis et al., 2018). The flat habitat at Heron Island had greater variability in both water temperature and light irradiance (van Oppen et al., 2018), and we found that flat genotypes had less partial mortality and higher experimental heat tolerance [measured as algal photosynthetic yield $(\mathrm{Fv} / \mathrm{Fm})]$ after transplantation to the slope compared to slope genotypes transplanted to the flat. This supports the general observation that individuals exposed to more variable environments are more tolerant and/or plastic, and thereby experience less stress when transplanted into more moderate environments (Smith et al., 2007; Barshis et al., 2010, 2013; Oliver and Palumbi, 2011; Kenkel and Matz, 2017). Another reciprocal transplant study reported a loss of heat tolerance in colonies transplanted to a more moderate environment (Palumbi et al., 2014), but here, though there was a slight reduction in heat tolerance for FS colonies, the FS and FF treatment groups' heat tolerance 18 months after transplantation was similar, revealing heat tolerance as an adaptation in the native flat population. Slope colonies transplanted to the flat had acclimatized in that they exhibited significantly greater heat tolerance compared to those that stayed at the slope. Thus, acclimatization or adaptation to a highly variable environment may promote survival and increased heat tolerance in coral populations.

The physiological performance of corals can be significantly affected by their Symbiodiniaceae community composition (Oliver and Palumbi, 2011; Palumbi et al., 2014; Howe-Kerr et al., 2019; Eckert et al., 2020). Here, we found that Symbiodiniaceae community composition was associated with the interaction of the amount of weight gained and transplant location at the Gardens site, and may also have contributed to heat tolerance. After 12 months of transplantation, two of three Gardens SF colonies were entirely comprised of C42 Symbiodiniaceae variants representative of the flat, one shuffled symbiont types to increase relative abundance of flat variants, and one had fixed flat C42 dominant community. Shuffling symbiont types in low abundance was also observed for one of three Canyons SF colonies. Minor bleaching in corals may allow hosts to obtain or increase optimal symbiont types to cope with environmental conditions (Baker, 2001; Berkelmans and van Oppen, 2006; Silverstein et al., 2015). The bleaching observed in most of the SF colonies after transplantation lead to high mortality overall, but also may have enabled the surviving colonies to successfully persist in a novel environment by shuffling algal symbionts. Sequence variant, C42.2, which was present in all flat-native and some slope-native colonies, was consistently found free-living in water samples taken at Heron Island reef flat habitats (Fujise et al., 2021), and points to the accessibility for uptake of C42 variants post-bleaching. Our results also point to the possible advantage of having a fixed symbiont community representative of a more variable environment as conditions continue to change. Some species within the genus Cladocopium are thought to be specialized to more variable or moderate habitats (Barshis et al., 2018; Eckert et al., 2020), and it is plausible that C42 variants may be attributed as specialized for species in the genus Pocillopora. C42 variants are repeatedly found in $P$. damicornis (van Oppen et al., 2018; Fujise et al., 2021) and were found to be dominant in $97 \%$ of Pocillopora grandis samples at Kiribati Island (Claar et al., 2020). Symbiodiniaceae variants that can withstand greater ranges in environmental conditions may subsequently increase resilience of the coral holobiont to environmental extremes.

Predictions of coral trait-related fitness in time and space require carefully designed field and lab experiments. Here, we show that replicate RTEs might yield differing population-byhabitat interactions, and other replicated experiments have also uncovered variation in physiological performance among sites (e.g., Drury and Lirman, 2021). The physiological differences between the Canyons and Gardens reef sites at Heron Island may be due to local environmental conditions such as hydrodynamic conditions and/or the coral tissue-associated prokaryote community. Both of the reef sites are located on the leeward side of the lagoon and are within a channel (Wistari Channel), but the Canyons site is located further southeast where the channel is wider and is relatively more exposed to southeastern waves. Due to the sharp reef crest found around Heron Island, waves coming from the southeast 
create wave-generated water flow over the reef flat in the same direction (Gourlay and Colleter, 2005). Further, the Canyons site has a more complex topography, the self-described underwater canyons create fingers of reef that stretch toward the reef edge, whereas the Gardens site has a straight shelf. The amount of water motion can affect the rate of gas and nutrient exchange between the coral tissue and the surrounding water (Dennison and Barnes, 1988), and the southeasterly water flow may flush through the underwater canyons, potentially causing the lower partial mortality seen in the Canyons FS treatment group. Minor differences in prokaryotic community composition between flat habitats at the Canyons and Gardens reef sites were previously reported (van Oppen et al., 2018), which may also have impacted physiological outcomes. Community composition of associated prokaryotes plays a role in the survival of Porites astroides colonies post-antibiotic treatment (Glasl et al., 2016) and Acropora digitifera colonies during heat stress (Gajigan et al., 2017), but were not measured here.

Genetically based variation in fitness is fuel for natural or artificial selection to promote performance under local prevailing conditions. Bleaching tolerance to thermal stress has a strong genetic basis in acroporid corals (Oliver and Palumbi, 2011; Howells et al., 2013; Bay and Palumbi, 2014; Dixon et al., 2015; Drury and Lirman, 2021) and Platygyra sp. (Elder et al., 2020), and here we extend knowledge to a representative of the genus Pocillopora. While bleaching tolerance is recognized to be polygenic (Fuller et al., 2020), host outlier loci may be used as biomarkers if they vary in individuals with differing performance to environmental conditions (Parkinson et al., 2020). Here, we could not detect variation in allelic or genotypic frequencies between surviving colonies in novel transplant treatment groups and their counterparts in their native habitat, likely because our sample sizes and experimental design lacked the power to examine this question with confidence, particularly if the trait is polygenetic and controlled by several genes of small effect. Single locus or polygenic DNA markers linked to performance (i.e., QTLs) are immensely useful to further study natural rates of adaptation and to identify stock for active seeding onto coral reefs. Our results highlight the additional importance of local environmental regimes and Symbiodiniaceae community to best describe variation in traits that affect fitness.

The active seeding of corals onto reefs is increasingly considered and applied in the management and conservation of these ecosystems (National Academies of Sciences, Engineering, and Medicine. (NAS), 2019; Bay et al., 2019). Seeded corals arise from a variety of methods (Randall et al., 2020) which may, or may not, consider the genetics of source stock (Baums et al., 2019). Selective breeding of standing genetic variation for heat tolerance has been proposed over latitudinal scales of the Great Barrier Reef (Dixon et al., 2015; Quigley et al., 2019). Here, we found extensive variation in traits that affect fitness, including heat tolerance of colonies among habitats separated by very short geographical distances. Brooding species make up 14\% of 444 Scleractinian species with known modes of sexual production (Harrison, 2011). Our results will likely be applicable for other brooding species, which are generally known to settle close to their parents, compared to spawning species (Ayre and Hughes, 2000). It is possible that seeding heat tolerant flat colonies to the slope habitat may assist in increasing tolerance in the slope population through gene flow of host putative adaptive loci and through maternal transmission of flat (C42) Symbiodiniaceae variants, as maternal transmission has been documented in P. damicornis (Epstein et al., 2019). The benefits of selecting coral stock for propagation and breeding must be balanced against potential risks (Aitken and Whitlock, 2013) including trade-offs among traits, as observed here. It should be recognized that applied reef restoration and adaptation studies use classic evolutionary experimental designs such as those presented here and thus will also further our fundamental understanding of microevolutionary processes of reef building corals under climate change.

\section{DATA AVAILABILITY STATEMENT}

The datasets presented in this study can be found in online repositories. The names of the repository/repositories and accession number(s) can be found below: NCBI PRJNA741589.

\section{AUTHOR CONTRIBUTIONS}

SM lead the project and compiled data, extracted DNA, ran statistics, interpreted data and wrote, and edited the manuscript. $\mathrm{KZ}$ was second leading advisor, helped analyze coral host outliers, and edited manuscript. JS was third leading advisor and edited manuscript. ML helped write code for statistics, interpret statistical results, and edited manuscript. MO designed experiment, field data collection, and edited manuscript. CK helped with data exploration and algal symbiont interpretation, and edited manuscript. LB was first leading advisor and PI of program that data was collected under, designed experiment, field data collection, and edited the manuscript. All authors contributed to the article and approved the submitted version.

\section{FUNDING}

The work was funded by the Australian Institute of Marine Science and AIMS@JCU. MO acknowledges Australian Research Council Laureate Fellowship FL180100036. CK acknowledges NSF International Postdoctoral Research Fellowship DBI1401165.

\section{ACKNOWLEDGMENTS}

We thank Ray Berkelmans for his key role in this project before his retirement from science. We also thank the crew on the RV Cape Ferguson, staff on Heron Island Research Station and numerous volunteers for their roles in the data collection. We are grateful to Carolyn Smith-Keune for granting temporary lab access, Veronique Mocellin for performing PCR on algal symbionts, Ben Hume for SymPortal analysis, Lesa Peplow for processing coral host genetic samples, and Pim Bongaerts for identifying coral host outliers. 


\section{SUPPLEMENTARY MATERIAL}

The Supplementary Material for this article can be found online at: https://www.frontiersin.org/articles/10.3389/fmars. 2021.704709/full\#supplementary-material

Supplementary Figure 1 | Absolute abundance of genotype frequencies of 16 single nucleotide polymorphism (SNP) outliers by habitat of surviving colonies combined by site after 12 months of reciprocal transplantation (March 2013). Native and foreign treatments are compared between source habitat by row, and between transplant habitat by column. Ref/ref = homozygote dominant, alt/alt $=$ homozygote alternate and ref/alt $=$ heterozygote.

Supplementary Table 1 | The number of colonies sampled in each treatment group and site used in the laboratory heat stress experiment 18 months after reciprocal transplantation in the field. Water was conditioned to temperature treatments at 24 (ambient), 29, 30 and $31^{\circ} \mathrm{C}$.

Supplementary Table 2 | The number of colonies sampled for Symbiodiniaceae community in coral tissue per site and treatment group at two time points. Native treatment groups were sampled in April 2012 and March 2013, and transplant treatment groups were sampled in March 2013.

Supplementary Table 3 | Partial mortality analysis of deviance table from Wald's Chi-square type III test on its generalized linear mixed model with data taken 18 months after reciprocal transplantation (August 2013). Bold values indicate statistical significance.

Supplementary Table 4 | Partial mortality pairwise contrasts based on estimated marginal means after 18 months of reciprocal transplantation (August 2013). Bold values indicate statistical significance.

Supplementary Table 5 | Weight gain analysis of deviance table from Wald's Chi-square type III test on its generalized linear mixed model with data taken 18 months after reciprocal transplantation (August 2013). Bold values indicate statistical significance.

Supplementary Table 6 | Weight gain pairwise contrasts based on estimated marginal means after 18 months of reciprocal transplantation (August 2013). Bold values indicate statistical significance.

Supplementary Table 7 | Heat stress analysis of deviance table from Wald's Chi-square type III test on the generalized linear mixed model of yield with data taken 18 months after reciprocal transplantation (August 2013). Bold values indicate statistical significance.

\section{REFERENCES}

Aitken, S. N., and Whitlock, M. C. (2013). Assisted gene flow to facilitate local adaptation to climate change. Annu. Rev. Ecol. Evol. Syst. 44, 367-388. doi: 10.1146/annurev-ecolsys-110512-135747

Anthony, K., Bay, L. K., Costanza, R., Firn, J., Gunn, J., Harrison, P., et al. (2017) New interventions are needed to save coral reefs. Nat. Ecol. Evol. 1:1420.

Ayre, D. J., and Hughes, T. P. (2000). Genotypic diversity and gene flow in brooding and spawning corals along the Great Barrier Reef, Australia. Evolution 54, 1590-1605. doi: 10.1554/0014-3820(2000)054[1590:gdagfi]2.0.co;2

Baker, A. C. (2001). Reef corals bleach to survive change. Nature 411, 765-766. doi: $10.1038 / 35081151$

Barshis, D. J., Birkeland, C., Toonen, R. J., Gates, R. D., and Stillman, J. H. (2018). High-frequency temperature variability mirrors fixed differences in thermal limits of the massive coral Porites lobata. J. Exp. Biol. 221:jeb188581.

Barshis, D. J., Ladner, J. T., Oliver, T. A., Seneca, F. O., Traylor-Knowles, N., and Palumbi, S. R. (2013). Genomic basis for coral resilience to climate change. Proc. Natl. Acad. Sci. U.S.A. 110, 1387-1392. doi: 10.1073/pnas.1210224110

Barshis, D. J., Stillman, J. H., Gates, R. D., Toonen, R. J., Smith, L. W., and Birkeland, C. (2010). Protein expression and genetic structure of the coral Porites lobata in an environmentally extreme Samoan back reef: does host genotype limit phenotypic plasticity? Mol. Ecol. 19, 1705-1720. doi: 10.1111/ j.1365-294x.2010.04574.x
Supplementary Table 8 | Heat stress pairwise contrasts based on estimated marginal means among different temperatures and contrasts of treatment groups. Bold values indicate statistical significance.

Supplementary Table 9 | Pairwise $F_{\mathrm{ST}}$ values (bottom diagonal) and $F_{\mathrm{ST}}$ $p$-values (top diagonal) between surviving colonies after 12 months of reciprocal transplantation (March 2013) for 16 single nucleotide polymorphism (SNP) outliers. Bold $p$-values indicate statistical significance. FF = flat to flat, $F S=$ flat to slope, $\mathrm{SF}=$ slope to flat, $\mathrm{SS}=$ slope to slope, $\mathrm{G}=$ Gardens site, $\mathrm{C}=$ Canyons site.

Supplementary Table 10 | Permutational multivariate analysis of variance of the Symbiodiniaceae composition in native treatment groups (FF, SS) between sampling time points in April 2012 and March 2013. Bold values indicate statistical significance.

Supplementary Table 11 | Permutational multivariate analysis of variance of the Symbiodiniaceae composition across source and transplant habitats (flat, slope) and sites (Canyons, Gardens) in March 2013 only. The combination of Source:Transplant refers to treatment group. Bold values indicate statistical significance.

Supplementary Table 12 | Permutational analysis of homogeneity of multivariate dispersions of the Symbiodiniaceae composition between site and treatment group combinations in March 2013 only.

Supplementary Table 13 | Pairwise permutational multivariate analysis of variance of Symbiodiniaceae community composition among site and treatment group comparisons in March 2013 only. Bold values indicate statistical significance.

Supplementary Table 14 | Permutational multivariate analysis of variance of the Canyons reef site Symbiodiniaceae community composition by source and transplant habitats (flat and slope) and physiological traits, percent partial mortality (PMORT) and percent cumulative weight gain (PCWEIGHT). Bold values indicate statistical significance.

Supplementary Table 15 | Permutational multivariate analysis of variance of the Gardens reef site Symbiodiniaceae community composition by source and transplant habitats (flat and slope) and physiological traits, percent partial mortality (PMORT) and percent cumulative weight gain (PCWEIGHT). Bold values indicate statistical significance.

Supplementary Table 16 | Matching genetic pair ID numbers used in the analysis of Symbiodiniaceae community composition and physiological traits shown in Figure 3.

Baums, I. B., Baker, A. C., Davies, S. W., Grottoli, A. G., Kenkel, C. D., Kitchen, S. A., et al. (2019). Considerations for maximizing the adaptive potential of restored coral populations in the western Atlantic. Ecol. Appl. 29:e01978.

Bay, L. K., Rocker, M., Bostrom-Einarsson, L., Buerger, P., Cleves, P., Harrison, D., et al. (2019). Reef Restoration and Adaptation Program: Intervention Technical Summary 3. URL https://gbrrestoration.org/wp-content/uploads/2020/09/T3Intervention-Technical-Summary-FINAL3.pdf (accessed July, 2020).

Bay, R. A., and Palumbi, S. R. (2014). Multilocus adaptation associated with heat resistance in reef-building corals. Curr. Biol. 24, 2952-2956. doi: 10.1016/j.cub. 2014.10.044

Bay, R. A., and Palumbi, S. R. (2017). Transcriptome predictors of coral survival and growth in a highly variable environment. Ecol. Evol. 7, 4794-4803. doi: 10.1002/ece3.2685

Berkelmans, R., and van Oppen, M. J. (2006). The role of zooxanthellae in the thermal tolerance of corals: a 'nugget of hope'for coral reefs in an era of climate change. Proc. R. Soc. B Biol. Sci. 273, 2305-2312. doi: 10.1098/rspb.2006.3567

Blanquart, F., Kaltz, O., Nuismer, S. L., and Gandon, S. (2013). A practical guide to measuring local adaptation. Ecol. Lett. 16, 1195-1205. doi: 10.1111/ele.12150

Blois, J. L., Zarnetske, P. L., Fitzpatrick, M. C., and Finnegan, S. (2013). Climate change and the past, present, and future of biotic interactions. Science 341, 499-504. doi: 10.1126/science. 1237184

Bongaerts, P., Riginos, C., Hay, K. B., van Oppen, M. J., Hoegh-Guldberg, O., and Dove, S. (2011). Adaptive divergence in a scleractinian coral: physiological 
adaptation of Seriatopora hystrix to shallow and deep reef habitats. BMC Evol. Biol. 11:303. doi: 10.1186/1471-2148-11-303

Brooks, M. E., Kristensen, K., Van Benthem, K. J., Magnusson, A., Berg, C. W., Nielsen, A., et al. (2017). glmmTMB balances speed and flexibility among packages for zero-inflated generalized linear mixed modeling. $R$ J. 9, 378-400. doi: 10.32614/rj-2017-066

Burrows, M. T., Schoeman, D. S., Buckley, L. B., Moore, P., Poloczanska, E. S., Brander, K. M., et al. (2011). The pace of shifting climate in marine and terrestrial ecosystems. Science 334, 652-655.

Chakravarti, L. J., Beltran, V. H., and van Oppen, M. J. (2017). Rapid thermal adaptation in photosymbionts of reef-building corals. Glob. Chang. Biol. 23, 4675-4688. doi: 10.1111/gcb.13702

Cheng, L., Zhu, J., Abraham, J., Trenberth, K. E., Fasullo, J. T., Zhang, B., et al. (2019). 2018 continues record global ocean warming. Adv. Atmos. Sci. 36, 249-252. doi: 10.1007/s00376-019-8276-x

Claar, D. C., McDevitt-Irwin, J. M., Garren, M., Vega Thurber, R., Gates, R. D., and Baum, J. K. (2020). Increased diversity and concordant shifts in community structure of coral-associated Symbiodiniaceae and bacteria subjected to chronic human disturbance. Mol. Ecol. 29, 2477-2491. doi: 10.1111/mec.15494

Dennison, W. C. and Barnes, D. J. (1988). Effect of water motion on coral photosynthesis and calcification. J. Exp. Mar. Biol. Ecol. 115, 67-77. doi: 10. 1016/0022-0981(88)90190-6

Dixon, G. B., Davies, S. W., Aglyamova, G. V., Meyer, E., Bay, L. K., and Matz, M. V. (2015). Genomic determinants of coral heat tolerance across latitudes. Science 348, 1460-1462. doi: 10.1126/science.1261224

Drury, C., and Lirman, D. (2021). Genotype by environment interactions in coral bleaching. Proc. R. Soc. B 288:20210177. doi: 10.1098/rspb.2021.0177

Eckert, R. J., Reaume, A. M., Sturm, A. B., Studivan, M. S., and Voss, J. D. (2020). Depth influences Symbiodiniaceae associations among Montastraea cavernosa corals on the Belize Barrier Reef. Front. Microbiol. 11:518. doi: 10.3389/fmicb. 2020.00518

Elder, H., Weis, V., Montalvo-Proano, J., Mocellin, V. J., Baird, A., Meyer, E., et al. (2020). Genetic variation in heat tolerance of the coral Platygyra daedalea offers the potential for adaptation to ocean warming. bioRxiv [Preprint]. doi: 10.1101/2020.10.13.337089

Ellegren, H., and Sheldon, B. C. (2008). Genetic basis of fitness differences in natural populations. Nature 452:169. doi: 10.1038/nature06737

Epstein, H. E., Torda, G., Munday, P. L., and van Oppen, M. J. (2019). Parental and early life stage environments drive establishment of bacterial and Dinoflagellate communities in a common coral. ISME J. 13, 1635-1638. doi: 10.1038/s41396019-0358-3

Excoffier, L., and Lischer, H. E. L. (2010). Arlequin suite ver 3.5: a new series of programs to perform population genetics analyses under Linux and Windows. Mol. Ecol. Res. 10, 564-567. doi: 10.1111/j.1755-0998.2010. 02847.x

Foll, M. (2012). BayeScan v2. 1 user manual. Ecology 20, 1450-1462.

Fujise, L., Suggett, D. J., Stat, M., Kahlke, T., Bunce, M., Gardner, S. G., et al. (2021). Unlocking the phylogenetic diversity, primary habitats, and abundances of free-living Symbiodiniaceae on a coral reef. Mol. Ecol. 30, 343-360. doi: $10.1111 / \mathrm{mec} .15719$

Fuller, Z. L., Mocellin, V. J., Morris, L. A., Cantin, N., Shepherd, J., Sarre, L., et al. (2020). Population genetics of the coral Acropora millepora: toward genomic prediction of bleaching. Science 369:eaba4674. doi: 10.1126/science.aba4674

Gajigan, A. P., Diaz, L. A., and Conaco, C. (2017). Resilience of the prokaryotic microbial community of Acropora digitifera to elevated temperature. MicrobiologyOpen 6:e00478. doi: 10.1002/mbo3.478

Galloway, L. F., and Etterson, J. R. (2007). Transgenerational plasticity is adaptive in the wild. Science 318, 1134-1136. doi: 10.1126/science.1148766

Glasl, B., Herndl, G. J., and Frade, P. R. (2016). The microbiome of coral surface mucus has a key role in mediating holobiont health and survival upon disturbance. ISME J. 10, 2280-2292. doi: 10.1038/ismej.2016.9

Gorospe, K. D., and Karl, S. A. (2015). Depth as an organizing force in Pocillopora damicornis: intra-reef genetic architecture. PLoS One 10:e0122127. doi: 10.1371/ journal.pone.0122127

Gourlay, M. R., and Colleter, G. (2005). Wave-generated flow on coral reefs-an analysis for two-dimensional horizontal reef-tops with steep faces. Coast. Eng. 52, 353-387. doi: 10.1016/j.coastaleng.2004.11.007
Hansen, J., Ruedy, R., Sato, M., and Lo, K. (2010). Global surface temperature change. Rev. Geophys. 48:RG4004.

Harrison, P. L. (2011). "Sexual reproduction of scleractinian corals," in Coral Reefs: An Ecosystem In Transition, eds Z. Dubinsky. and N. Stambler. (Dordrecht: Springer), 59-85. doi: 10.1007/978-94-007-0114-4_6

Henn, J. J., Buzzard, V., Enquist, B. J., Halbritter, A. H., Klanderud, K., Maitner, B. S., et al. (2018). Intraspecific trait variation and phenotypic plasticity mediate alpine plant species response to climate change. Front. Plant Sci. 9:1548. doi: 10.3389/fpls.2018.01548

Hoegh-Guldberg, O., Poloczanska, E. S., Skirving, W., and Dove, S. (2017). Coral reef ecosystems under climate change and ocean acidification. Front. Mar. Sci. 4:158. doi: $10.3389 /$ fmars. 2017.00158

Hoeksema, J. D., and Forde, S. E. (2008). A meta-analysis of factors affecting local adaptation between interacting species. Am. Nat. 171, 275-290. doi: 10.1086/ 527496

Howe-Kerr, L., Bachelot, B., Wright, R. M., Kenkel, C. D., Bay, L. K., and Correa, A. M. (2019). Symbiont community diversity is more constrained in holobionts that tolerate diverse stressors. bioRxiv, 572479 [Preprint].

Howells, E. J., Berkelmans, R., van Oppen, M. J., Willis, B. L., and Bay, L. K. (2013). Historical thermal regimes define limits to coral acclimatization. Ecology 94, 1078-1088. doi: 10.1890/12-1257.1

Hughes, T. P., Baird, A. H., Bellwood, D. R., Card, M., Connolly, S. R., Folke, C., et al. (2003). Climate change, human impacts, and the resilience of coral reefs. Science 301, 929-933. doi: 10.1126/science.1085046

Hume, B. C., Smith, E. G., Ziegler, M., Warrington, H. J., Burt, J. A., LaJeunesse, T. C., et al. (2019). SymPortal: a novel analytical framework and platform for coral algal symbiont next-generation sequencing ITS2 profiling. Mol. Ecol. Res. 19, 1063-1080. doi: 10.1111/1755-0998.13004

Kawecki, T. J., and Ebert, D. (2004). Conceptual issues in local adaptation. Ecology Lett. 7, 1225-1241. doi: 10.1111/j.1461-0248.2004.00684.x

Kenkel, C. D., and Matz, M. V. (2017). Gene expression plasticity as a mechanism of coral adaptation to a variable environment. Nat. Ecol. Evol. 1:0014.

Lenth, R., Singmann, H., Love, J., Buerkner, P. and Herve, M. (2019). 2019 emmeans: Estimated Marginal Means, Aka Least-Squares Means.

Marshall, D. J. (2008). Transgenerational plasticity in the sea: context-dependent maternal effects across the life history. Ecology 89, 418-427. doi: 10.1890/070449.1

McMurdie, P. J., and Holmes, S. (2013). phyloseq: an R package for reproducible interactive analysis and graphics of microbiome census data. PloS One 8:e61217. doi: 10.1371/journal.pone.0061217

National Academies of Sciences, Engineering, and Medicine. (NAS) (2019). A Decision Framework For Interventions To Increase The Persistence And Resilience Of Coral Reefs. Washington, WA: National Academies Press.

Oksanen, J., Blanchet, F. G., Friendly, M., Kindt, R., Legendre, P., McGlinn, D., et al. (2019). Package "vegan". R package version. 2.5-6.

Oliver, T. A., and Palumbi, S. R. (2011). Do fluctuating temperature environments elevate coral thermal tolerance? Coral Reefs 30, 429-440. doi: 10.1007/s00338011-0721-y

Palumbi, S. R., Barshis, D. J., Traylor-Knowles, N., and Bay, R. A. (2014). Mechanisms of reef coral resistance to future climate change. Science 344, 895-898. doi: 10.1126/science.1251336

Parkinson, J. E., Baker, A. C., Baums, I. B., Davies, S. W., Grottoli, A. G., Kitchen, S. A., et al. (2020). Molecular tools for coral reef restoration: beyond biomarker discovery. Conserv. Lett. 13, e12687.

Parmesan, C., and Yohe, G. (2003). A globally coherent fingerprint of climate change impacts across natural systems. Nature 421:37. doi: 10.1038/ nature 01286

Prada, C., and Hellberg, M. E. (2013). Long prereproductive selection and divergence by depth in a Caribbean candelabrum coral. Proc. Natl. Acad. Sci.U.S.A. 110, 3961-3966. doi: 10.1073/pnas.1208931110

Price, N. N., Muko, S., Legendre, L., Steneck, R., van Oppen, M. J. H., Albright, R., et al. (2019). Global biogeography of coral recruitment: tropical decline and subtropical increase. Mar. Ecol. Prog. Ser. 621, 1-17. doi: 10.3354/meps 12980

Putnam, Hollie M, and Gates, Ruth D. (2015). Preconditioning in the reefbuilding coral Pocillopora damicornis and the potential for trans-generational acclimatization in coral larvae under future climate change conditions. J. Exp. Biol. 218, 2365-2372. doi: 10.1242/jeb.123018 
Quigley, K. M., Bay, L. K., and van Oppen, M. J. (2019). The active spread of adaptive variation for reef resilience. Ecol. Evol. 9, 11122-11135. doi: 10.1002/ ece3.5616

Quigley, K. M., Bay, L. K., and Willis, B. L. (2018). Leveraging new knowledge of Symbiodinium community regulation in corals for conservation and reef restoration. Mar. Ecol. Prog. Ser. 600, 245-253. doi: 10.3354/meps12652

R Core Team (2020). R: A language and environment for statistical computing. R Foundation for Statistical Computing. Vienna: R Core Team. URL https: //www.R-project.org/ (accessed February, 2020).

Randall, C. J., Negri, A. P., Quigley, K. M., Foster, T., Ricardo, G. F., Webster, N. S., et al. (2020). Sexual production of corals for reef restoration in the Anthropocene. Mar. Ecol. Prog. Ser. 635, 203-232. doi: 10.3354/meps 13206

Savolainen, O., Lascoux, M., and Merilä, J. (2013). Ecological genomics of local adaptation. Nat. Rev. Genet. 14:807. doi: 10.1038/nrg3522

Siebeck, U. E., Marshall, N. J., Klüter, A., and Hoegh-Guldberg, O. (2006). Monitoring coral bleaching using a colour reference card. Coral Reefs 25, 453-460. doi: 10.1007/s00338-006-0123-8

Silverstein, R. N., Cunning, R., and Baker, A. C. (2015). Change in algal symbiont communities after bleaching, not prior heat exposure, increases heat tolerance of reef corals. Glob. Chang Biol. 21, 236-249. doi: 10.1111/gcb.12706

Smale, D. A. (2020). Impacts of ocean warming on kelp forest ecosystems. New Phytol. 225, 1447-1454. doi: 10.1111/nph.16107

Smith, L. W., Barshis, D., and Birkeland, C. (2007). Phenotypic plasticity for skeletal growth, density and calcification of Porites lobata in response to habitat type. Coral Reefs 26, 559-567. doi: 10.1007/s00338-007-0216-z

Takabayashi, M., and Hoegh-Guldberg, O. (1995). Ecological and physiological differences between two colour morphs of the coral Pocillopora damicornis. Mar. Biol. 123, 705-714. doi: 10.1007/bf00349113

Torda, G., Donelson, J. M., Aranda, M., Barshis, D. J., Bay, L., Berumen, M. L., et al. (2017). Rapid adaptive responses to climate change in corals. Nat. Clim. Chang. 7:627. doi: $10.1038 /$ nclimate3374

Van Hooidonk, R., Maynard, J., Tamelander, J., Gove, J., Ahmadia, G., Raymundo, L., et al. (2016). Local-scale projections of coral reef futures and implications of the Paris Agreement. Sci. Rep. 6, 1-8. van Oppen, M. J., Bongaerts, P., Frade, P., Peplow, L. M., Boyd, S. E., Nim, H. T., et al. (2018). Adaptation to reef habitats through selection on the coral animal and its associated microbiome. Mol. Ecol. 27, 2956-2971. doi: 10.1111/mec. 14763

Wernberg, T., Bennett, S., Babcock, R. C., De Bettignies, T., Cure, K., Depczynski, M., et al. (2016). Climate-driven regime shift of a temperate marine ecosystem. Science 353, 169-172. doi: 10.1126/science.aad8745

West, J. M., Harvell, C. D., and Walls, A. M. (1993). Morphological plasticity in a gorgonian coral (Briareum asbestinum) over a depth cline. Mar. Ecol. Prog. Ser. 94:61. doi: 10.3354/meps094061

Wilson, K., Li, Y., Whan, V., Lehnert, S., Byrne, K., Moore, S., et al. (2002). Genetic mapping of the black tiger shrimp, Penaeus monodon with amplified fragment length polymorphism. Aquaculture 204, 297-309. doi: 10.1016/s0044-8486(01) 00842-0

Ziegler, M., Seneca, F. O., Yum, L. K., Palumbi, S. R., and Voolstra, C. R. (2017). Bacterial community dynamics are linked to patterns of coral heat tolerance. Nat. Commun. 8:14213.

Conflict of Interest: The authors declare that the research was conducted in the absence of any commercial or financial relationships that could be construed as a potential conflict of interest.

Publisher's Note: All claims expressed in this article are solely those of the authors and do not necessarily represent those of their affiliated organizations, or those of the publisher, the editors and the reviewers. Any product that may be evaluated in this article, or claim that may be made by its manufacturer, is not guaranteed or endorsed by the publisher.

Copyright (c) 2021 Marhoefer, Zenger, Strugnell, Logan, van Oppen, Kenkel and Bay. This is an open-access article distributed under the terms of the Creative Commons Attribution License (CC BY). The use, distribution or reproduction in other forums is permitted, provided the original author(s) and the copyright owner(s) are credited and that the original publication in this journal is cited, in accordance with accepted academic practice. No use, distribution or reproduction is permitted which does not comply with these terms. 